\title{
Périurbain et tram-train : enjeux, projets et réalisations
}

Joël Forthoffer

\section{OpenEdition}

1 Journals

Édition électronique

URL : https://journals.openedition.org/rge/3615

DOI : $10.4000 /$ rge.3615

ISSN : 2108-6478

Éditeur

Association des géographes de l'Est

Édition imprimée

Date de publication : 15 juin 2012

ISSN : 0035-3213

\section{Référence électronique}

Joël Forthoffer, « Périurbain et tram-train : enjeux, projets et réalisations », Revue Géographique de l'Est [En ligne], vol. 52 / 1-2 | 2012, mis en ligne le 04 février 2013, consulté le 21 septembre 2021. URL:

http://journals.openedition.org/rge/3615 ; DOI : https://doi.org/10.4000/rge.3615

Ce document a été généré automatiquement le 21 septembre 2021.

Tous droits réservés 


\title{
Périurbain et tram-train : enjeux, projets et réalisations
}

\author{
Joël Forthoffer
}

1 Dans les dernières décennies, les villes ont changé d'échelle. Elles s'étalent de plus en plus loin de leurs centres et de leurs banlieues. Le renchérissement des coûts de logement en zone centrale, le déplacement des activités vers leur périphérie, le développement d'infrastructures routières et l'utilisation de la voiture sont autant de raisons qui expliquent la délocalisation de l'habitat. Mais la périurbanisation s'est faite sans souci de cohérence le long des axes de communications, autoroutes et voies rapides. Philippe Hamman (2011) fait état « d'une pluralité d'échelles urbaines et à leur mise en connexion, au niveau de l'agglomération ou de la métropole, et non uniquement du centre ville». L'engorgement routier, les difficultés de stationnement, la pollution et les nuisances liées à la voiture amènent les autorités organisatrices à rechercher des solutions de transports collectifs pour répondre à ces marchés.

2 Le développement de ces espaces façonnés par l'automobile, et généralement mal desservis par les transports publics, engendre un recours accru à son utilisation, entraînant l'aggravation de la congestion des voies d'accès aux centres villes. Aujourd'hui, ce marché représente près de $38 \%$ des déplacements en province. On connaît son importance en Île-de-France (2,7 millions de voyageurs transportés chaque jour sur Transilien). Il est en pleine expansion, comme le montrent toutes les études sur les déplacements au sein des agglomérations. Dans le cadre de politiques globales visant à réduire ou contenir l'usage de la voiture, il faut trouver des réponses en matière de transports publics aux questions de dessertes des zones périphériques.

3 C'est ainsi que l'existence d'étoiles ferroviaires autour des agglomérations devrait constituer une perspective pour une desserte en transports collectifs. L'étude qui suit présente des solutions nouvelles envisagées pour adapter l'offre de transport en commun en site propre à certaines aires urbaines en France et dans l'espace rhénan. 


\section{Périurbain et transports collectifs}

4 L'espace périurbain est en dehors de la ville, mais en même temps il entretient des rapports étroits avec elle. Ainsi, en s'appuyant sur l'évolution des modes de transports, et en allant s'installer à l'extérieur des centres urbains, les habitants cherchent à recréer à la campagne les caractéristiques sociales et matérielles des villes. Ces espaces d'interpénétrations de la ville et de la campagne se caractérisent par d'importantes migrations au quotidien, entre le domicile et le lieu de travail, avec un développement en périphérie d'un habitat pavillonnaire. Les espaces périurbains sont liés à la très forte mobilité des populations et à l'accroissement de la distance domicile - travail.

5 La périurbanisation et l'extension des aires d'influence des villes ne sont pas une spécificité française. En Allemagne comme en Suisse, les principales agglomérations ont aussi vu leurs périphéries se développer dès les années 1960. Cette extension entraîne des besoins nouveaux et nécessite d'étendre l'offre de transport collectif. La figure $n^{\circ} 1$ représente les évolutions des déplacements et modélise l'intégration des transports dans les espaces pour une meilleure planification.

Figure $\mathrm{n}^{\circ} 1$ : Evolution des déplacements urbains et périurbains.

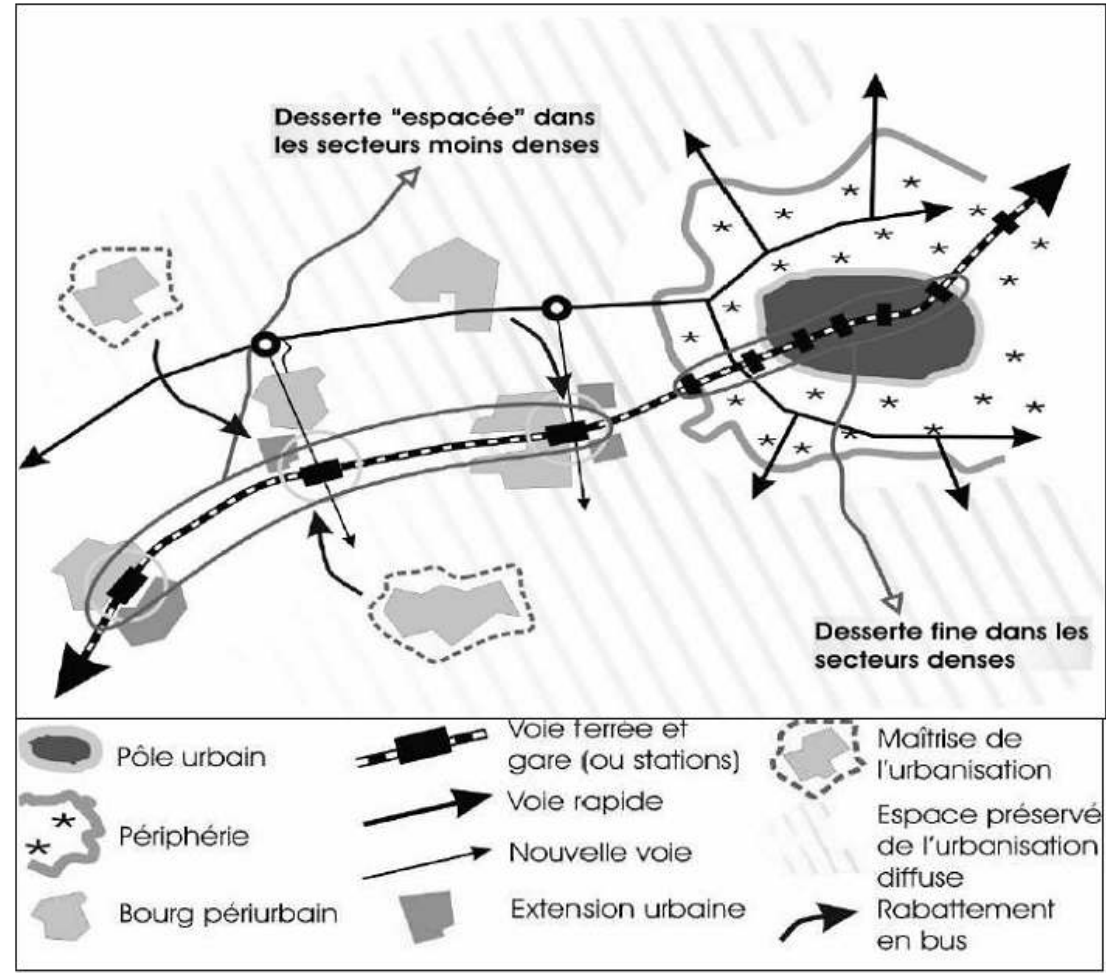

Source : Projet Bahn - Ville (2003)

En Suisse au début des années 70, après la réduction des investissements consacrés à l'infrastructure ferroviaire, apparaissent les problèmes de saturation sur la route. L'essor économique contribue à l'explosion du volume du trafic de transit avec des traversées alpines qui sont surchargées. Les régions marquées par une forte densité de la population sont elles aussi saturées. Les Suisses prennent conscience des limites de 
cette évolution et des perspectives qu'offre le chemin de fer . En 1987, ils mettent en place le projet «Rail 2000».

7 En collaboration avec d'autres entreprises de transport et avec les cantons, les Chemins de Fer Fédéraux ont mis au point le concept des Réseau Express Régionaux (RER), dont les limites correspondent approximativement à celles des huit plus grandes agglomérations suisses : Zurich, St Gall, Bâle, Suisse Centrale, Genève, Vaud, Tessin et Bern. Le premier RER a été mis en service à Zurich en 1990 avec une desserte cadencée, des lignes traversant le centre ville, du matériel moderne et performant (accès facilité aux trains), des tarifs et informations homogènes... Dans ce schéma l'intermodalité avec les transports collectifs urbains, bus, tramway ou métro s'effectue dans les gares. A partir de 2005, la cadence semi-horaire devient la norme sur la plupart des lignes en Suisse. Aux heures de pointe, les trains de certaines lignes circulent à des fréquences encore plus rapprochées.

8 Parmi ces agglomérations figurent également Bâle. Si les premières réflexions sur la mise en place d'un réseau express trinational remontent à 1980, il faudra attendre la fin des années 1990 pour voir s'étendre le réseau bâlois vers Mulhouse (F) puis vers Freiburg im Breisgau (D). Le projet «RER Région de Bâle 2005 » avec ses composantes horaires, matériels roulants et infrastructures propose un cadencement à la demiheure sur toutes les lignes avec intervalles réguliers, uncadencement au quart d'heure au sein de la région ${ }^{1}$. Aujourd'hui le réseau RER de Bâle exploite près de $250 \mathrm{~km}$ de lignes et acheminent près de 20 millions de personnes par an. Un réseau tramway urbain et tramway régional avec des pôles d'échanges complètent le dispositif.

9 Ainsi les transports publics en Suisse privilégient l'interconnexion des différents modes de transports collectifs. La présence d'une communauté de transport et d'une communauté tarifaire sont la norme, facilitent l'intermodalité avec les moyens existants sans la recherche systématique de nouvelles technologies tels que le tramtrain (Beyer cité dans Hamman, 2011).

10 Fort de leurs expériences dans les travaux de restructuration des graphiques horaires, les cabinets de consultants suisses sont appelés aux chevets des Länder allemands dans les années 1990. En dehors de Berlin (1930) il aura fallu attendre les années 1960 pour de timides développements des S-Bahn (abréviation pour "(Stadt)schnellbahn»). Ce n'est qu'après les années 1990 que d'autresagglomérations se dotent d'un système de SBahn interconnecté à un réseau de tramway ou de métro (U-Bahn). Ces transports express se caractérisent par des horaires cadencés et denses, des stations proches les unes des autres, une tarification intermodale, une intermodalité avec les autres moyens de transports et pour de nombreux réseaux la traversée du centre ville en tunnel. La desserte ferroviaire des zonespériurbaines et rurales s'ajoute à la desserte urbaine plutôt avancée en comparaison du système français, en raison de la plus forte densité du territoire allemand et des orientations différentes choisies par les autorités organisatrices de transports.

11 Le Land Rhénanie-Palatinat avec la S-Bahn Regio RheinNeckar compte parmi eux, suivi de près par le Land Bade-Wurtemberg. Dans un premier temps, il a fallu estimer les investissements nécessaires en infrastructure des lignes existantes, comme des lignes nouvelles, pour établir un cadencement horaire ou semi horaire. Aujourd'hui, la S-Bahn RheinNeckar avec son réseau de 290 kilomètres forme l'épine dorsale du réseau de transport ferroviaire de la région métropolitaine avec les villes de Ludwigshafen, Mannheim, Heidelberg, Kaiserslautern et Karlsruhe. 
12 C'est dans ce contexte que Karlsruhe, agglomération de 265000 habitants, lance en 1992 la première ligne entre jusqu'à Bretten. Ce système de transport interconnecté permet à du matériel roulant polyvalent de circuler tant sur des voies du tramway que de chemin de fer. Appelé tramway hybride («Zweisystem-Stadbahn »), puis tram-train, le « modèle de Karlsruhe » se caractérise par trois principes :

13 - la possibilité du matériel roulant de circuler en mixité avec d'autres matériels (tramway et trains) sur chacun des réseaux ;

14 - l'absence de rupture de charge qui permet d'assurer la continuité du transport entre centre ville et périphérie ;

15 - la mise en connexion des réseaux pour assurer le passage effectif du matériel roulant d'un réseau à l'autre.

$16 \mathrm{Au}$ fil des ans, l'agglomération de Karlsruhe, avec les réseaux de tram-train de Karlsruhe et Heilbronn, le tramway urbain et suburbain a constitué un réseau de transport collectif qui s'apparente à un S-Bahn ou « mini RER » selon François Enver (p. 30, Spécial Tram-Train, Ville et Transports $n^{\circ} 423$ ).

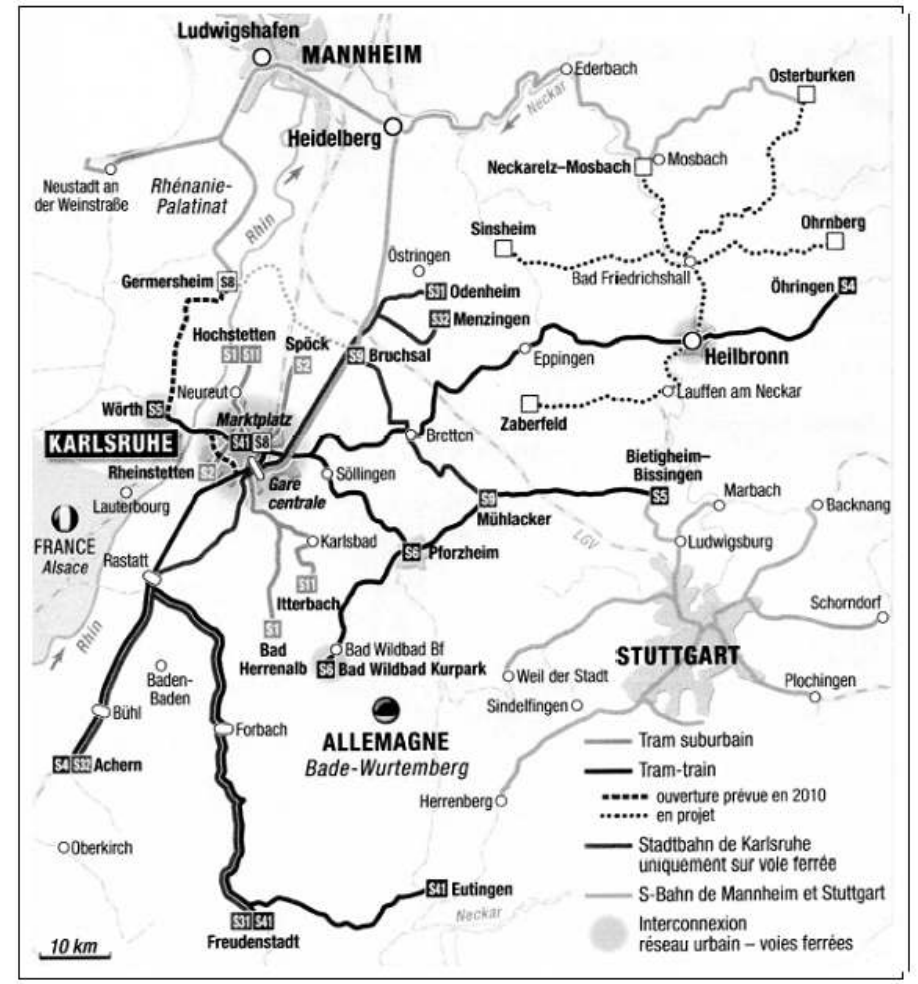

Figure $\mathrm{n}^{\circ} 2$ : Desserte régionale de Karlsruhe, une forte présence du tram-train

Source : d'après Géographie des transports (2005)

17 Le terme tram-train est souvent utilisé à tort pour désigner des projets de tramway express ou de tramway régional ou de tramway périurbain. A titre d'exemple, la ligne Aulnay-Bondy (Ile de France, 2006) ou Nantes-Clisson (Pays de Loire, 2010) circulent dans leur phase actuelle sur des voies de réseau ferré mais sans interconnexion avec le réseau urbain de tramway. Le RhôneExpress qui relie l'aéroport de Saint-Exupéry à la gare de Lyon Part-Dieu circule sur des voies réservées, sans interconnexion avec le réseau urbain et sur une section de ligne en mixité avec un tramway urbain. 
Photographie $n^{\circ} 1$ : Parvis gare de Lyon La Part Dieu ; Rhône express et tramway urbain (Joël Forthoffer - 14 mars 2012)



Tableau $n^{\circ} 1$ : Synthèse des réseaux tram - train étudiés

\begin{tabular}{|c|c|c|c|c|c|c|}
\hline $\begin{array}{c}\text { Ville } \\
\text { (Région) }\end{array}$ & $\begin{array}{l}\text { Population } \\
\text { Ville } \\
\text { Agglomération } \\
\text { (Nb hab) }\end{array}$ & $\begin{array}{c}\text { Année } \\
\text { de } \\
\text { mise en } \\
\text { service }\end{array}$ & \begin{tabular}{|c|} 
Réseau \\
Tram train \\
$\left(\begin{array}{c}\text { Nb ligne }) \\
(\mathrm{km})\end{array}\right.$ \\
\end{tabular} & $\begin{array}{c}\text { Matériel } \\
\text { Nb rames } \\
\text { motorisation }\end{array}$ & Exploitant & Observations \\
\hline $\begin{array}{l}\text { Karlsruhe } \\
\text { (B. } \\
\text { Württemberg) }\end{array}$ & $\begin{array}{l}291000 \\
700000\end{array}$ & 1992 & $\begin{array}{c}7 \\
347 \mathrm{~km}\end{array}$ & $\begin{array}{c}121 \\
\text { bifréquence }\end{array}$ & $\begin{array}{c}\text { Abtal } \\
\text { Verkehrs } \\
\text { Gesellschaft } \\
\text { mbH } \\
\end{array}$ & $\begin{array}{l}\text { Vers la création } \\
\text { d'un S Bahn }\end{array}$ \\
\hline $\begin{array}{l}\text { Saarbrücken } \\
\text { (Saarland) }\end{array}$ & $\begin{array}{l}177000 \\
340000\end{array}$ & 1997 & $\begin{array}{c}1 \\
18 \mathrm{~km}\end{array}$ & $\begin{array}{c}28 \\
\text { bifréquence }\end{array}$ & $\begin{array}{l}\text { StadtbahnSaar } \\
\text { GmbH }\end{array}$ & \\
\hline $\begin{array}{l}\text { Kassel } \\
\text { (Hessen) }\end{array}$ & $\begin{array}{l}196000 \\
450000\end{array}$ & 2007 & $\begin{array}{c}4 \\
122 \mathrm{~km}\end{array}$ & $\begin{array}{c}28 \\
\text { bifréquence } \\
\text { diesel électrique }\end{array}$ & $\begin{array}{c}\text { KVG } \\
\& \\
\text { DB Regio }\end{array}$ & \\
\hline $\begin{array}{l}\text { Mulhouse } \\
\text { (Alsace) }\end{array}$ & $\begin{array}{l}112000 \\
255000\end{array}$ & 2010 & $\begin{array}{c}1 \\
22 \mathrm{~km}\end{array}$ & $\begin{array}{c}12 \\
\text { bifréquence }\end{array}$ & $\begin{array}{l}\text { Soléa } \\
\& \text { SNCF }\end{array}$ & $\begin{array}{l}2^{\text {ème }} \text { phase } \\
\text { prévue } \\
\text { prolongement } \\
\text { Kruth }\end{array}$ \\
\hline $\begin{array}{l}\text { Nantes } \\
\text { (Pays de Loire) }\end{array}$ & $\begin{array}{l}290000 \\
550000\end{array}$ & 2011 & $\begin{array}{c}1 \\
36 \mathrm{~km}\end{array}$ & $\begin{array}{c}24 \\
\text { bifréquence }\end{array}$ & SNCF & $\begin{array}{l}2^{\text {mme }} \text { phase en } \\
\text { cours : } 64 \mathrm{~km} \\
\text { Nantes- } \\
\text { Chateaubriand }\end{array}$ \\
\hline
\end{tabular}

Source : études et rapports d'activités

\section{Les acteurs et les freins à la mise en œuvre du tram-train}

Dans un contexte d'utilisation croissante des transports publics, de réduction des frontières entre l'urbain et le périurbain, il s'agit de développer des concepts innovants : adaptés aux nouveaux modes de vie, le tram-train répond aux exigences 
d'intermodalité, de simplicité et de fluidité des déplacements. Solution originale, il peut aussi bien circuler sur le réseau ferroviaire, comme untrain classique, que pénétrer au coeur des villes, à la manière d'un tramway. Il permet ainsi l'accès direct au centre ville sans changement de mode. Il dessert en outre plus de stations qu'un train dans le même temps, grâce à ses capacités d'accélération et de freinage supérieurs.

Ce système de transport permet de relier les réseaux de tramway urbain et les réseaux ferroviaires situés dans la périphérie des villes. Après les premières expériences et la première génération de systèmes d'exploitation mixtes en Allemagne (Karlsruhe et Saarbrück), nous assistons actuellement à la naissance de nouveaux réseaux.

L'engouement pour le tram-train au milieu des années 1990 se solde par un nombre impressionnant d'études de faisabilité visant à vérifier la pertinence du système pour de nombreuses villes européennes. Beaucoup de villes et de régions disposant d'un réseau ferroviaire régional, avec ou sans réseau de tramway, de taille identique à la ville de Karlsruhe, réfléchissent alors à la possibilité d'adapter les principes d'exploitation mixte à leur contexte.

21 L'étude de ces réseaux ( $\mathrm{cf}$. tableau ${ }^{\circ} 1$ ) montre que les conditions de déplacements entre centre ville et périphérie deviennent de plus en plus difficiles. Malgré le développement de leurs services, les transports urbains restent gênés par le manque de liaisons entre les réseaux de chemins de fer régionaux ou de banlieue et les tramways ou métros légers. A l'inverse quand la gare est éloignée du centre ville, les trains ne peuvent pas pénétrer au cœur du tissu urbain, faute d'infrastructure. Ce qui explique qu'il y a quelques années encore, les zones d'actions respectives des autorités organisatrices des transports urbains et des transports interurbains étaient nettement délimitées. Mais au quotidien, les déplacements de type domicile-travail, domicileétudes effectués en transports collectifs impliquent dans presque tous les cas, l'utilisation d'au moins deux modes de transport pour rejoindre le centre ville.

Aussi les enjeux du transport collectif périurbain prennent une autre dimension. Il s'agit d'harmoniser dans une agglomération les différents réseaux et l'intermodalité avec les flux de déplacements et transports. Cette solution innovante proposée aux autorités organisatrices supprime tout " effet frontière " entre les transports de la périphérie et ceux de la ville, entre le "périurbain » et l'«urbain ». Le tram-trainest en lui même un système de transport intermodal dont lesprincipales caractéristiques sont reprises dans le tableau $n^{\circ} 2$ ci-après. 
Tableau $n^{\circ} 2$ : La souplesse du tramway, la performance du train

\begin{tabular}{|c|c|c|}
\hline $\begin{array}{c}\text { TRAMWAY } \\
+\end{array}$ & $\begin{array}{c}\text { TRAIN } \\
=\end{array}$ & TRAM - TRAIN \\
\hline $\begin{array}{l}\Leftrightarrow \text { horaires et fréquences de } \\
\text { passage en adéquation avec les } \\
\text { besoins de déplacement } \\
\Leftrightarrow \text { desserte fine du territoire } \\
\text { urbain avec de nombreuses } \\
\text { stations } \\
\Leftrightarrow \vec{c} \text { accès facilité pour tous les } \\
\text { voyageurs } \\
\Leftrightarrow \text { vitesse plus élevée que le bus } \\
\Leftrightarrow \text { plus économique qu'un } \\
\text { métro }\end{array}$ & $\begin{array}{l}\Leftrightarrow \rightarrow \text { confort de voyage } \\
\Leftrightarrow \text { rame plus grande que } \\
\text { bus/tramway/métro, donc plus de } \\
\text { places offertes } \\
\Leftrightarrow \text { vitesse de pointe plus élevée } \\
\text { qu'un tramway } \\
\Leftrightarrow \text { ponctualité dans les horaires } \\
\text { de départ et d'arrivée } \\
\Leftrightarrow \text { départs et arrêts se font depuis } \\
\text { une gare }\end{array}$ & $\begin{array}{l}\Leftrightarrow \text { voyage sans rupture de } \\
\text { charge entre la périphérie et le } \\
\text { centre ville } \\
\Leftrightarrow \text { maillage fin du territoire } \\
\Leftrightarrow \text { mobilité durable de bout en } \\
\text { bout } \\
\Leftrightarrow \text { associe la rapidité du train en } \\
\text { réseau périurbain et la fluidité } \\
\text { du tramway en réseau urbain }\end{array}$ \\
\hline
\end{tabular}

Face à l'engorgement des métropoles, les collectivités locales ont compris que le tramtrain conjuguait l'effet positif du tramway dans le périmètre urbain et celui du ferroviaire traditionnel dans un secteur allant jusqu'à quelques dizaines de kilomètres du centre-ville. Très attractif pour les voyageurs, il leur fait gagner du temps sur leur parcours. En évitant les ruptures de charge, c'est un facteur d'induction de trafic majeur qui fait diminuer la contribution publique au voyageur. D'où l'intérêt des collectivités territoriales à opter pour ce mode de transport. Mais le coût d'investissement pour les infrastructures reste un frein même si l'on utilise généralement une partie des infrastructures existantes. Il en est de même du surcoût du matériel roulant lié aux équipements de sécurités et d'alimentation. Ainsi, le coût d'exploitation reste élevé pour les collectivités et au final, le coût du tram-train demeure un frein à son expansion.

En tant que système de transport à la fois urbain et périurbain ou même régional, les projets tram-train impliquent un grand nombre d'acteurs dont les logiques et les intérêts ne sont pas toujours convergents. A la croisée des compétences transport de l'agglomération, du département et de la région, ces projets posent la question de l'imbrication entre le périmètre de transport urbain (PTU) qui relève de l'autorité organisatrice urbaine et les transports régionaux qui sont gérés par le conseil régional. Ces projets intègrent nécessairement une dimension urbanistique rajoutant des acteurs (villes, bureaux d'urbanisme, Etat...) et des niveaux de planification territoriale supplémentaires. Enfin, ces projets nécessitent une coopération entre les exploitants avec des questions centrales à régler sur le partage des pouvoirs et des risques avec la répartition géographique et technique (conduite, maintenance du matériel) des responsabilités d'exploitation, mais également des charges et des recettes. Le tramtrain conduit à ré-inventer tous les services aux voyageurs pour s'harmoniser plus encore : tarification des billets, coordination des systèmes billettique, organisation et modalité du contrôle...

De plus il a conduit à de nouveaux modes de partenariats, avec des AO inédites (les villes, les agglomérations, les communautés d'agglomérations... selon les cas) et des transporteurs urbains. Que se soit en Allemagne ou en France, cette diversité d'acteurs, $\mathrm{du}$ fait de leurs intérêts divergents, ne se prête pas toujours à l'émergence de consensus. D'où des stratégies d'acteurs, des jeux d'influences et de positionnement exercés aux différents échelons du pouvoir de décision. 
26 A titre d'exemple, le tram-train qui Mulhouse à la vallée de la Thur illustre bien la collaboration des nombreux acteurs au-delà des aspects financiers. Une première réflexion a parmi d'établir une comparaison entre l'agglomération et l'aire périurbaine de Mulhouse avec celle de Karlsruhe. Possédant des configurations et des structures de population comparable, le volontarisme des acteurs politiques évoque le projet dès 1990. Le tram-train, dont le terme a été proposé en 1999 par Jean-Marie Bockel, alors député-maire de Mulhouse, a été pensé dès le début en parallèle avec le projet de tramway de Mulhouse (mis en service en 2006). Puis l'étroite collaboration entre les autorités organisatrices, Région Alsace et Mulhouse Alsace Agglomération (M2A) qui ont pris la suite du Sitram ont permis de lancer le projet dès 1996, en partenariat avec le Conseil Général du Haut-Rhin. Déclaré d'utilité publique en octobre 2004, l'ensemble des partenaires du projet a décidé en novembre 2005 de lancer la première phase opérationnelle jusqu'à Thann caractérisé par la réalisation de l'interconnexion des réseaux ferroviaire et urbain, l'acquisition du matériel et la mise en œuvre d'une desserte mixte assurée par des trains régionaux et des tram-trains grâce aux quatre kilomètres de voie nouvelle dédiée (Mairelle, 2010, p.15).

27 La réalisation est le fruit d'un long travail impliquant tous les partenaires qui participent au financement en fonction de leur domaine de compétence :

28 - l'Etat, qui a soutenu le projet par le plan de relance et financé dans le cadre du contrat de projets Etat Région,

29 - les Autorités Organisatrices de Transports qui définissent l'offre de service et la tarification, financent le matériel roulant, le coût d'exploitation et participent à l'infrastructure,

30 - Réseau Ferré de France, maître d'ouvrage des travaux d'infrastructure ferroviaire (4 $\mathrm{km}$ de voie nouvelle entre Mulhouse et Lutterbach),

31 - la SNCF, propriétaire des gares, qui équipe les points d'arrêts en mobilier et système d'information. Elle a également supervisé l'achat des 12 rames et piloté la mise au point des trams - trains avec l'industriel Siemens (démarches agrément auprès des instances de sécurité),

32 - Soléa (groupe Transdev-Véolia) et la SNCF, respectivement exploitants du réseau urbain de Mulhouse et TER Alsace, ont noué un partenariat innovant pour la mise en œuvre du service (conduite mutualisée, gestion des circulations, maintenance, information voyageurs...). 
Tableau $n^{\circ} 3$ : Bilan du plan de financement

\begin{tabular}{|l|r|r|r|r|r|r|r|}
\hline \multicolumn{1}{|c|}{ Millions d'euros } & Total & Etat & RFF & SNCF & CG68 & m2A & Région Alsace \\
\hline Infrastructures & 84,400 & 27,440 & 7,600 & 0,975 & 10,550 & 7,974 & 29,861 \\
\hline $\begin{array}{l}\text { Matériel roulant } \\
\text { tram-train }\end{array}$ & 52,88 & & & & & 19,19 & 33,69 \\
\hline Atelier-dépôt & 9,77 & & & & & 3,59 & 6,18 \\
\hline Total & & $\begin{array}{r}\mathbf{1 8 , 7 \%} \\
\mathbf{2 7 , 4 4 0}\end{array}$ & $\begin{array}{r}\mathbf{5 , 2 \%} \\
\mathbf{7 , 6 0 0}\end{array}$ & $\begin{array}{r}\mathbf{0 , 6 \%} \\
\mathbf{0 , 9 7 5}\end{array}$ & $\begin{array}{r}\mathbf{7 , 2} \% \\
\mathbf{1 0 , 5 5 0}\end{array}$ & $\begin{array}{r}\mathbf{2 0 , 9 \%} \\
\mathbf{3 0 , 7 5 4}\end{array}$ & $\begin{array}{r}\mathbf{4 7 , 4 \%} \\
\mathbf{6 9 , 7 3 1}\end{array}$ \\
\hline
\end{tabular}

Source : dossier de presse SNCF (2010)

Un comité de pilotage constitué de ces partenaires a également été mis en place dès 2000 , lancé les études puis débattu et arbitré les questions complexes de la tarification multimodale, la formation et l'habilitation des conducteurs sur l'ensemble du parcours, la maintenance de l'infrastructure et du matériel.

Les autorités organisatrices s'entendent pour la mise en œuvre d'un service de transport collectif reposant sur la complémentarité des modes de transport: bus, tramway, tram-train, train et autocars. Elles demandent aux exploitants qu'ils développent et facilitent l'intermodalité en se coordonnant.

L'ensemble de ces démarches a permis la mise en service commerciale le 12 décembre 2011. Et Guillaume Pépy d'annoncer lors de l'inauguration: «En réalisant et en exploitant de façon innovante l'offre de service tram-train, SNCF poursuit son engagement de construire avec les collectivités locales, un transport public au service du développement économique, social et culturel des territoires. En gommant la frontière entre l'urbain et le périurbain, l'offre de service tram-train participe à cet objectif » et Hubert du Mesnil, Président Directeur Général de Réseau Ferré de France, de souligner que « le tram-train Mulhouse Vallée de la Thur connecte deux réseaux de transport dotés chacun au départ de spécificités propres. Cette innovation est le fruit d'une volonté politique territoriale forte et d'un partenariat exemplaire ».

Ainsi, au moment du renouveau du tramway et des trains légers, il y a vingt-cinq ans environ, les services ferroviaires régionaux étaient, en comparaison, dans un mauvais état. Des infrastructures délabrées, un matériel roulant anciens et des lignes qui fermaient. Le passage de dessertes ferroviaires classiques à des dessertes de type tramtrain, telles que celle de Karlsruhe-Bretten ou Saarbrücken-Sarreguemines, a représenté un renouveau. Cependant, que se soit en Allemagne ou en France, de nombreux projets tram-train ne dépassent le stade d'étude d'avant-projet en raison de la complexité des dossiers et la présence d'acteurs multiples qui peinent à s'entendre et trouver les financements.

\section{Des réalisations et des projets}

Le périurbain est un segment du marché dans lequel la croissance est très forte compte tenu de l'étalement de l'urbanisation. Il représente donc pour les exploitants un potentiel de développement important. En évitant au voyageur l'intermodalité entre 
l'urbain et le ferroviaire avec les délais de correspondances, l'augmentation de la fréquentation des services tram-train peut être particulièrement massive. Cette possibilité de croissance n'existe probablement que dans l'univers favorable au rail (déplacements radiaux dans un corridor desservi par une voie ferrée), suppose souvent des investissements importants (infrastructures, matériel roulant, équipements, billettique) et un renforcement de l'offre (cadencement horaire).

Les besoins des clients périurbains sont très spécifiques: prédominance des déplacements pendulaires aux heures de pointe, ce qui implique cadencement, fluidité, intermodalité, accessibilité des gares, information en temps réel, qualité et fiabilité. Ces attentes font peser des exigences fortes sur les modèles de production. Il apparaît très nettement que la concurrence sur le périurbain viendra des opérateurs urbains.

Pour permettre à une même rame de circuler sur des voies de tramway en centre-ville et de relier des stations situées en périphérie, le matériel utilisé doit être compatible avec le chemin de fer classique (signalisation, puissance, résistance). L'offre de ce mode de transport en commun contribue à un maillage plus efficace de l'ensemble du réseau, notamment en cas de combinaison avec le tramway classique. Très développé dans les pays germaniques, ce système a été mis partiellement en service en 2006, en région parisienne avec la réutilisation de l'ancienne ligne des Coquetiers entre Bondy et Aulnay-sous-Bois. Au sens strict de la définition du tram-train, en France, la première ligne ouverte est celle du tram-train Mulhouse-Vallée de la Thur qui depuis le 12 décembre 2010 relie Mulhouse à Thann.

Figure $n^{\circ} 3$ : Un système complexe et évolutif

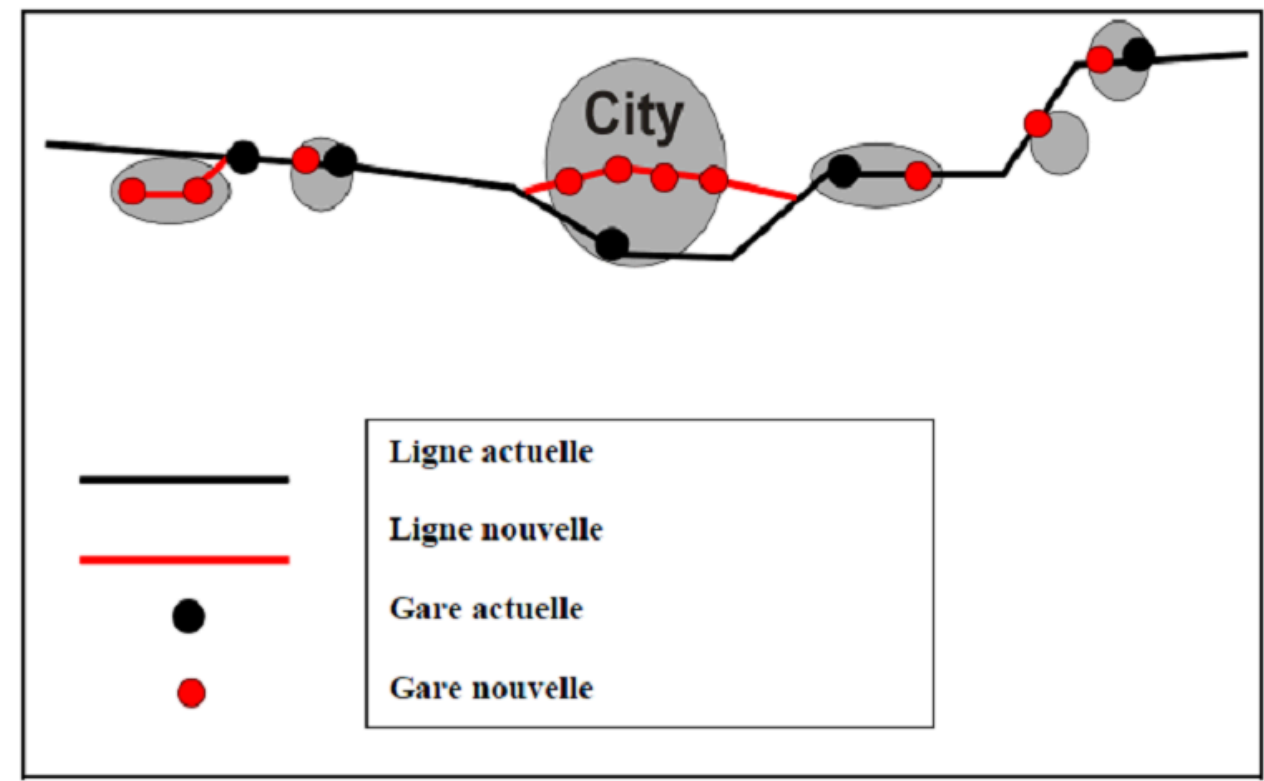

Source : Joël Forthoffer (2011)

Le tramway interurbain est d'une certaine manière un train parce que le matériel roulant répond aux normes de sécurité pour circuler sur une ligne ferroviaire, mais il également est utilisé en service urbain. Il circule sur une ligne ferroviaire classique comme un train de banlieue et éventuellement, les plans prévoient des rails au centreville pour faire une partie de son trajet comme un tramway. 
41 Les projets de tram-train sont en général plus compliqués à mettre en oeuvre que d'autres projets de transport en commun en site propre. Ils réclament par là même un niveau élevé d'organisation, de contrôle et de qualité. Presque tous les projets englobent des corridors régionaux. L'implication des institutions régionales ou d'institutions équivalentes ainsi que le soutien politique sont des conditions de base de la réussite. C'est pourquoi le sénateur Haenel préconisait la création d'une autorité organisatrice unique (p. 11, Les transports ferroviaires périurbains, Le rail nº17/18).

La définition d'objectifs clairs et convaincants, lors de la phase de planification, est un aspect primordial pour la réussite du projet dont les procédures de planification de transport sont complexes. Plus que pour tout autre projet, les réflexions liées à la mise en place d'une ligne sont liées aux choix en terme d'urbanisme et d'occupation des sols. La hiérarchie urbaine et l'analyse de la structure territoriale sont également des éléments à prendre en compte. Une structure régionale centralisée sera un avantage d'autant plus important pour la réussite d'un projet de tram-train que l'attractivité de sa capitale sera importante. A l'inverse, une structure d'agglomération multipolaire ne favorise pas les projets de lignes directes vers le centre ville.

Karlsruhe: succès, crises et faiblesses. Au départ, la société d'exploitation urbaine souhaitait étendre sa zone de desserte hors de la ville avec la ligne de Bretten pour répondre aux besoins des voyageurs. Aujourd'hui, dans le monde entier, le nom de Karlsruhe est synonyme de succès en matière de transport public. Pourtant son système dispose d'un certain nombre de caractéristiques qu'il serait difficile de trouver ou de transposer ailleurs. C'est certainement un bon exemple de ville pour laquelle la distance entre la gare centrale et le centre ville est importante. Ceci augmente naturellement l'attractivité d'une liaison de transport public directe entre la périphérie et le centre ville. Avec un réseau très marqué régionalement (cf. figure $\mathrm{n}^{\circ} 2$ ), comprenant plusieurs lignes de tram-train traversant le centre ville, il montre aujourd'hui ses limites (M. Casazza, Verbundsbericht Geschaftsjahr 2009). Les autorités sont aujourd'hui confrontées à un nouveau problème de transport avec la saturation des lignes en centre ville. Elles cherchent à le résoudre grâce à la création d'un tunnel dans l'artère principale du réseau, ce qui impliquerait également la fin des circulations de tramways classiques dans la zone piétonne. Malgré cela, les augmentations de fréquentation du réseau de Karlsruhe ont confirmé l'intérêt de liaisons directes et continues des centres villes dont la gare est excentrée. Mais paradoxalement, ce succès a également rappelé l'intérêt des gares centrales qui facilitent la desserte urbaine. 
Photographie $n^{\circ} 2$ : tram-train parvis de la gare de Karsruhe, ligne 4 Karlsruhe Heilbronn (Joël Forthoffer - 25 août 2011)

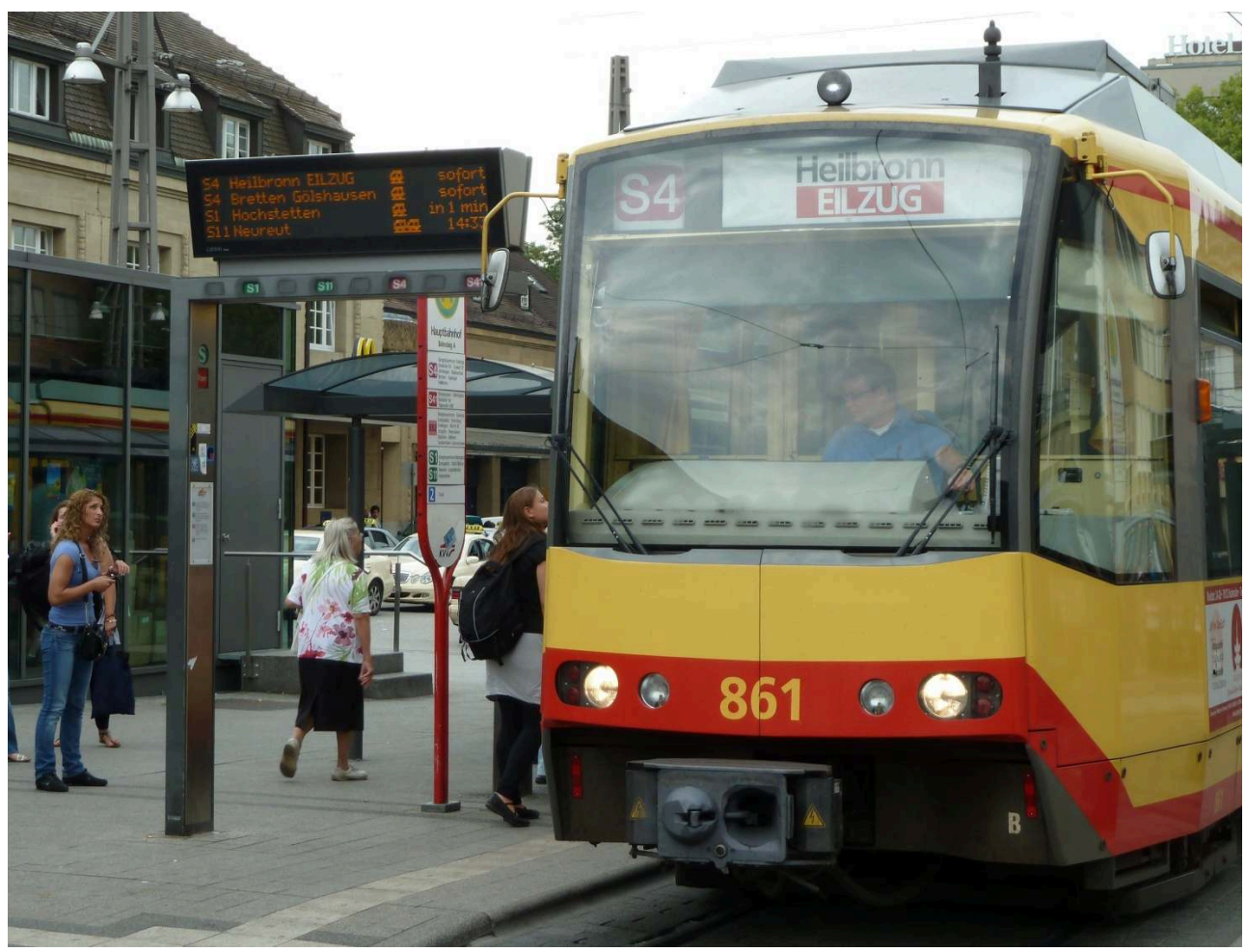

Saarbrücken : le premier tram-train à plancher bas.En raison d'un réseau ferroviaire dense, lié au développement historique de l'industrie du charbon et de l'acier et l'absence de ligne de tramway classique dont l'exploitation a été arrêtée en 1965, une solution de type tram-train à exploitation mixte a été choisie en 1991 par la ville de Saarbrücken. Dès 1997, la première ligne relie le centre à la ville française de Sarreguemines grâce à la réalisation de $5 \mathrm{~km}$ de lignes réalisées en mode tramway à travers les rues de la ville jusqu'à Brebach, puis par l'emprunt des $13 \mathrm{~km}$ de voies ferrées. L'existence du tracé de la voie ferrée DB a fortement contribué à cette réalisation.La rapidité d'exécution et de réalisation, aussi bien des études que des travaux, n'a été possible que grâce à un climat politique favorable marqué par une grande coopération entre les acteurs de la ville et de la région. Contrairement à Karlsruhe, le constructeur du matériel Bombardier-Eurorail a développé dès le départ un système à plancher bas intégral. L'absence de réseau de tramway urbain a facilité la résolution des problèmes techniques qui avaient fait l'objet de nombreux compromis à Karlsruhe. Par la suite la ligne a été prolongée d'environ $6 \mathrm{~km}$ en direction de Riegelsberg vers le nord-ouest.

Photographie $n^{\circ} 3$ : Tram-train entrant en gare de Sarreguemines (Joël Forthoffer 25 août 2011) 


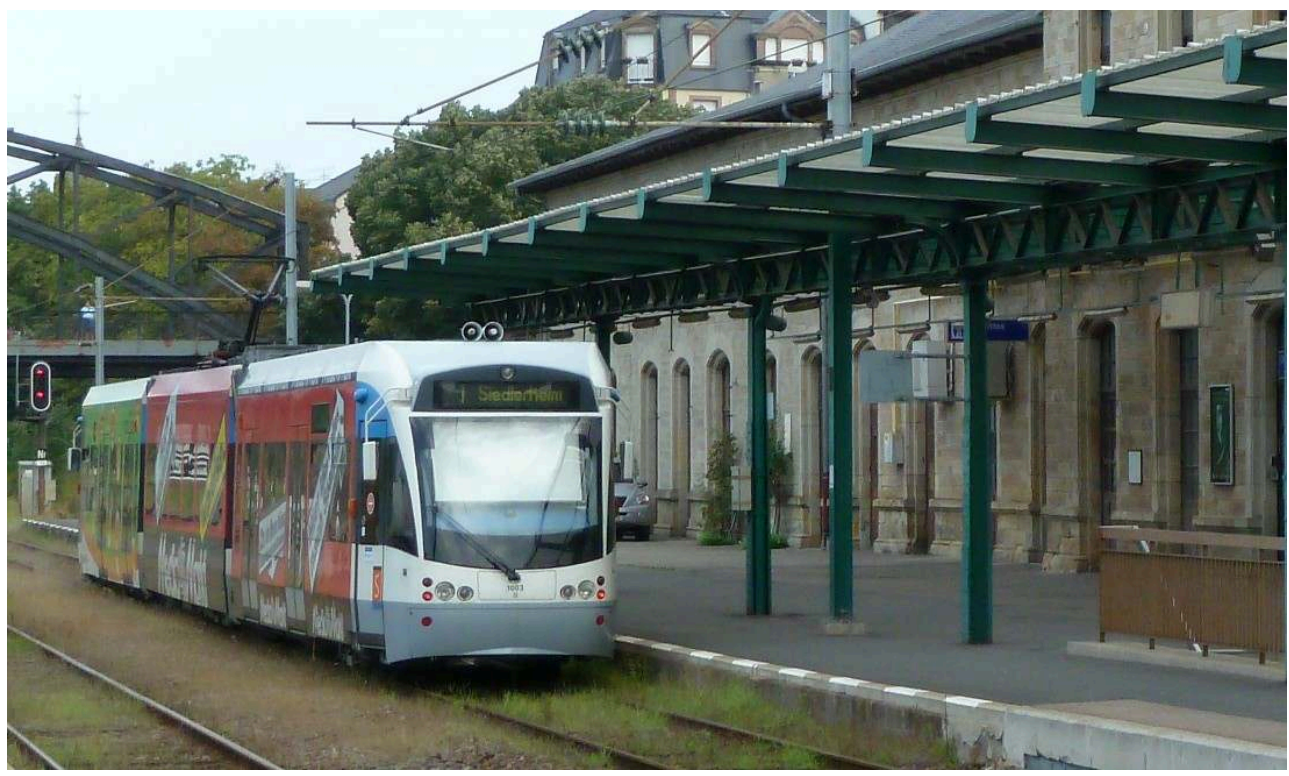

Les exemples de Karlsruhe et Saarbrücken montrent l'importance du phasage dans le développement et la réalisation d'un réseau. En Allemagne, les transports urbains ne sont pas soumis systématiquement à la mise en concurrence et les procédures d'appel d'offre ont facilité l'extension des réseaux des exploitants au-delà du périmètre urbain. Pendant cette décennie des années 1990, il faut également noter que le peu d'intérêt marqué par la DB, opérateur ferroviaire historique, a facilité leur réalisation. D’autres projets n'ont pas connu le même sort et ont été abandonnés dans les premières phases d'étude, ajournés ou déclinés en tramway régional. Les raisons sont multiples et diverses. Elles vont du manque de volonté politique aux conditions structurelles ou législatives non favorables (règles de consultations), en passant par des difficultés techniques qui entrainent des surcoûts.

Le RegioTram de Kassel mis en service en 2007 relève d'une exploitation en coopération entre DB Regio et la société de transport Regional Bahn Kassel. La gare centrale de Kassel en cul-de-sac avec la mise en place d'une nouvelle ligne à grande vitesse Frankfurt-Hannover-Berlin voit son importance diminuer au bénéfice de la gare de Kassel Wilhelmshöhe située sur cette ligne. La réalisation d'une interconnexion entre le réseau ferroviaire et le réseau urbain permet aux tram -train de passer sous le bâtiment voyageur par l'intermédiaire d'une rampe (cf. photographie). Les travaux d'aménagement ont débuté en 2004 (95 M€) et permettent aujourd'hui d'exploiter quatre lignes qui répondent au concept du tram-train. Au-delà des performances techniques et économiques, l'aboutissement du projet repose sur la coopération des différents acteurs: les autorités organisatrices des transports, la communauté de transport et les différentes activités de DB (Netz AG, Station \& Service AG, Energie). DB Regio qui participe à l'exploitation (p. 23 Sylvain Zalkind dans Transports Urbains $n^{\circ} 119$ ) montre ainsi son intérêt pour les transports suburbains et se positionne ainsi sur ces marchés qu'il ne souhaite laisser aux seules mains des opérateurs urbains qui étaient les initiateurs des premiers tram-train en Allemagne. 
Photographie $n^{\circ} 4$ : Le RegioTram de Kassel rejoint le réseau ferré DB par un tunnel (Joël Forthoffer - Kassel 18 août 2011)



En France, il faut attendre les années 2000 pour voir apparaître les premières réalisations. Mise en service en novembre 2006 en Ile-de-France, la ligne reliant Bondy à Aulnay-sous-Bois constitue une première réponse aux besoins du marché des déplacements périurbains sans répondre à tous les critères d'un tram-train puisque la ligne ferroviaire n'est empruntée que pour rejoindre le dépôt. Mais avec cette première étape, la SNCF marque son intérêt pour le concept de tram-train (p. 95, Cécile Lière, Tramways, Revue Générale des Chemins de Fer $n^{\circ} 170$ ).

Le tram-train Mulhouse-Vallée de la Thur, inspiré du modèle de Karlsruhe et motivé par le désengorgement du nœud ferroviaire de Mulhouse et une desserte plus dense, a été mis en service le 11 décembre 2010 avec dans un premier temps, une desserte entre Mulhouse et Thann. Son exploitation est assurée en coopération par le transporteur urbain, la société Soléa et la SNCF. Son principal objectif est de devenir une alternative à la voiture. Cette nouvelle offre de service entre l'agglomération mulhousienne et la vallée de la Thur repose sur la complémentarité entre les différents modes de transport: train régional, tram-train, tramway, car interurbain et bus. Les points d'arrêts desservis sont conçus comme des zones d'échanges avec des connexions facilitées vers les autres modes de transport tels que le train, les bus, le vélo. Des amplitudes horaires élargies, des dessertes cadencées et une tarification commune, innovante et attractive favorisent par ailleurs l'utilisation quotidienne de ce nouveau mode de transport. 
Photographie $n^{\circ} 5$ : Présentation officielle du tram-train de Mulhouse-Thann (Raymond Woessner9 décembre 2009)



Le tram-train Pays de Loire avec la ligne Nantes-Clisson a été mis en service en juin 2011 avec trois aller retour par jour et un objectif fixé par la Région à l'horizon 2014 : un tram-train toutes les trente minutes aux heures de pointe. Son objectif est de densifier les services. Lors de son inauguration, le président de région Pays de Loire, Jacques Auxiette, confirme cette première étape en vue d'un véritable réseau: « $\mathrm{Ce}$ tram-train ne circule pas sur les voies du tramway de Nantes mais il est compatible et il permettra éventuellement un jour de faire un embranchement ». Après la rénovation de la ligne, le tram-train doit arriver à Chateaubriand situé à une soixantaine de kilomètres de Nantes au printemps 2013 et au futur aéroport de Notre-Dames-desLandes en $2017 \ldots$ 
Photographie $n^{\circ} 6$ : tram-train de Nantes en instance de départ gare de Clisson (Joël Forthoffer - 15 juillet 2011)

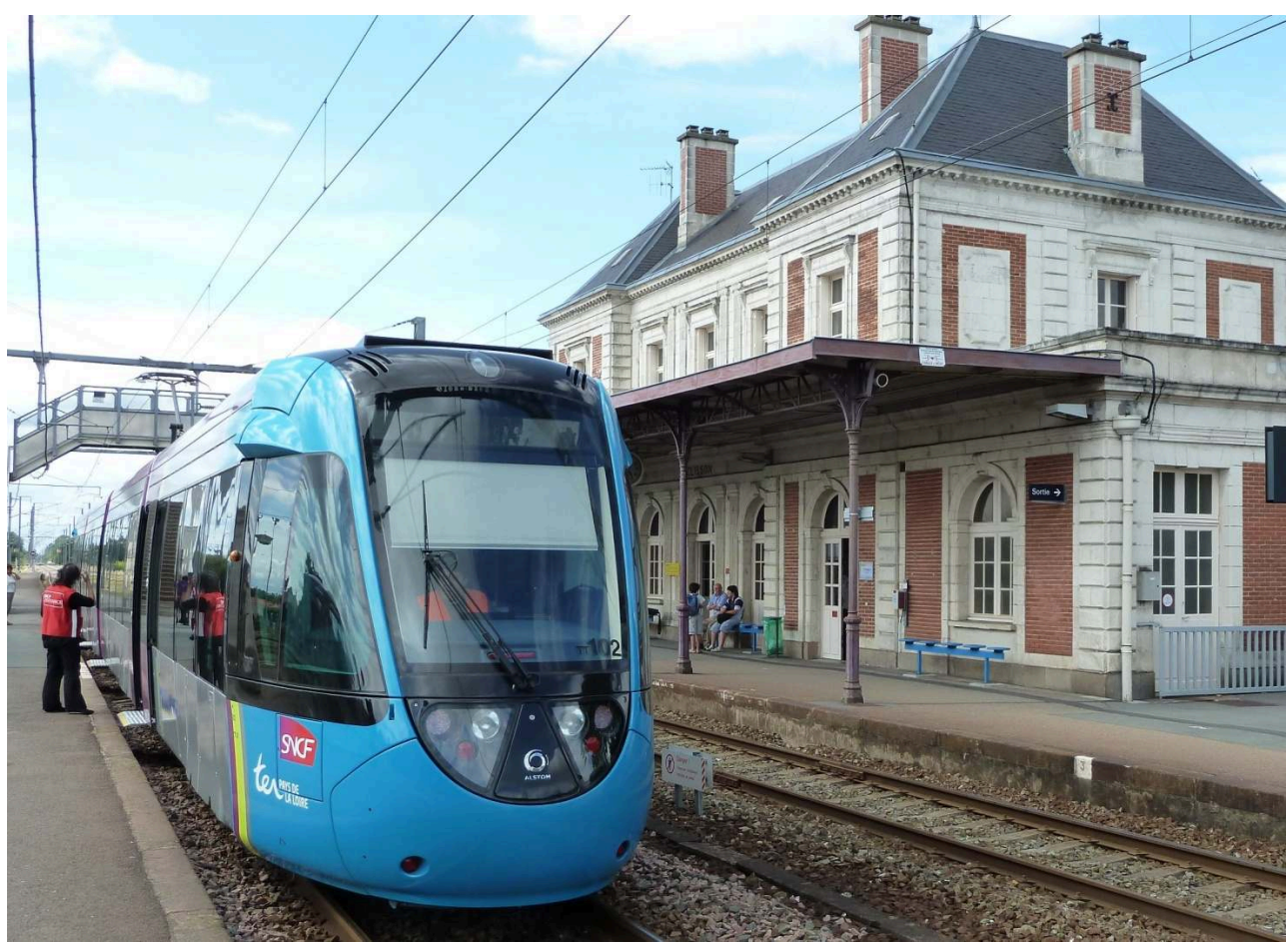

51 La standardisation du matériel roulant et les engagements pris par les différents acteurs aux projets participent au développement du tram-train en France. En effet, en 2006, la Région Rhône-Alpes a initié une démarche fédérative menée avec la Région Pays de la Loire et le Groupement des Autorités Responsables de Transport (GART). Cette démarche impliquant plusieurs Régions a conduit la SNCF, en charge de la maitrise d'œuvre, à lancer un appel d'offre pour la construction d'un tram-train après l'analyse des besoins, l'estimation de la taille du marché et l'élaboration du cahier des charges. La mutualisation des besoins des régions influe sur le volume des rames potentielles. Il permet ainsi des économies d'échelles significatives grâce à des offres plus attractives de la part des constructeurs. L'appel d'offre porte sur un marché potentiel de 200 rames (tranche ferme et optionnelle) dont une tranche ferme de 31 rames à fournir par l'industriel retenu dès 2009. Les 31 rames se répartissent en : 24 rames pour l'Ouest Lyonnais et 7 rames pour Nantes - Châteaubriant. Les tranches optionnelles concernent 169 rames dont 10 rames supplémentaires pour Pays de Loire, 10 rames supplémentaires pour Rhône-Alpes, 83 rames pour l'Ile-de-France et 14 rames pour l'Alsace. Le constructeur Alstom a été retenu pour la construction du tram-train nouvelle génération et le livre depuis 2010. Ces commandes traduisent-elles les seules perspectives de développements en Allemagne et en France pour les années à venir?

\section{Conclusion}

Depuis la fin des années 1990, beaucoup d'autorités organisatrices urbaines et régionales considèrent, à l'image de Karlsruhe, le tram-train comme la solution miracle. Cependant, le nombre de projets réalisés reste limité et nombreux sont ceux qui ont été modifiés en cours d'étude. Même en Allemagne, où les conditions réglementaires et politiques sont les plus propices au développement de tels systèmes, 
l'évolution ne se fait que très lentement au regard des potentiels existants. Quelques projets devraient se concrétiser en France à l'horizon 2015-2020, à Strasbourg avec la desserte de Bruche Piémont des Vosges, à Lyon (Ouest Lyonnais), à Nantes avec NantesChâteaubriant (Pays de Loire), et en Ile-de-France avec des lignes tangentielles légères du Transilien.

53 S'il est difficile de trouver une explication unique à cette situation, nous pouvons, sur la base des projets étudiés, apporter quelques éléments de réponse. Le développement du tram-train comme solution périurbaine interconnecté est fortement liés aux contextes locaux (institutionnels, culturels, techniques,...) qui sont très inégaux ou font souvent défaut. Si l'intégration des réseaux est souvent possible techniquement, l'intégration des organisations, des règles et des procédures se révèlent souvent complexes à organiser dans un environnement où la concurrence prime sur le partenariat, d'où le sort nuancé des différents projets qui ont suivi. Les récents intérêts de la part des gestionnaires de réseaux, qui y trouvent des solutions pour désengorger les nœuds ferroviaires aux abords des agglomérations, tout comme des opérateurs ferroviaires historiques, qui ne souhaitent laisser ces marchés aux mains des opérateurs urbains, vont-ils contribuer à leur essor?

Malgré les nombreuses réflexions, en dehors des revues techniques et scientifiques, il existe peu d'ouvrages qui traitent de façon exhaustive du sujet, sans doute à l'image des projets non réalisés.

\section{BIBLIOGRAPHIE}

BAVOUX J.J., BEAUCIRE F., CHAPELON L., ZEMBRI P. (2005), « Géographie des transports », Armand Colin, collection U, Paris, $231 \mathrm{p}$.

CASAZZA W. (2009), Verbundbericht Jahr 2006 - 2011, Karlsruhe, 32 p.

FORTHOFFER J. (2011), Milieu périurbain et mobilités par les transports en commun dans le Rhin supérieur, Les cahiers de l'Association de Prospective Rhénane, 168, pp. 26-38.

HAMMAN P. (2011), «Le tramway dans la ville - le projet urbain négocié à l'aune des déplacements », Presses universitaires de Rennes, coll. « Espace et territoire », Rennes, 288 p.

MAIRELLE H. (2010), « Regards partagés sur le tram-train Mulhouse Vallée de la Thur », Editions Carré Blanc, Strasbourg, 68 p.

\section{Revues Scientifiques:}

Le Rail HS n¹7/18, Les transports ferroviaires périurbains, novembre 1999, 68 p.

Revue Générale des Chemins de Fer n 170, Tramways, mars 2008, 142 p.

Transports Urbains n¹19, Tram-Train et territoire, novembre 2011, 38 p.

Ville \& Transports Magazine n423, Spécial Tram-Train, 25 avril 2007, 68 p. 


\section{NOTES}

1. Le cadencement horaire en Suisse est développé dans un autre article (Forthoffer, 2011).

\section{RÉSUMÉS}

Depuis près d'un demi-siècle déjà, les agglomérations connaissent un développement important de leur périphérie qui entraîne des besoins croissants de mobilité. Face à cette situation et dans un contexte de contraintes budgétaires, des acteurs publics ont pris la décision de mettre en place de nouveaux systèmes de transports en commun en site propre comme le tram-train. Il s'agit de la connexion d'un réseau existant de chemin de fer régional à celui d'un métro léger ou tramway pour offrir aux passagers des liaisons directes de transports entre le centre ville et la périphérie. Si la liaison de tramway Karlsruhe - Bretten en 1992 marque la première mise en service, entre temps nous notons l'émergence de nombreux projets... et de quelques réalisations. Dans ce contexte de dynamique des territoires périurbains, cette communication s'interroge sur le rôle des acteurs (politiques, bureau d'études, AOT, exploitants, constructeurs,...) et s'intéresse aux preneurs de décisions. Elle se propose d'examiner cette problématique en s'appuyant sur des exemples concrets. Tout d'abord, à partir des caractéristiques et spécificités des réalisations des techniques tram-train, nous examinons le processus de leur mise en place, sans oublier l'analyse de ceux restés à l'état de projets. Ensuite, nous nous intéressons plus spécifiquement aux nombreux acteurs et à leur coopération pour répondre aux besoins de coordinations. Enfin, face aux enjeux des territoires, il est intéressant d'observer quelles seront les perspectives de développement.

For nearly half a century, the cities are developing important to their periphery resulting in growing demand for travel. Faced with this situation and in a context of budget constraints, public actors have decided to set up new systems for public transit in dedicated lanes such as tram-train. This is the connection of an existing network of regional railway to that of a light rail or tram to offer passengers direct connections between the transportation center and the periphery.

If tram link Karlsruhe-Bretten in 1992, marks the first time in the meantime we note the emergence of many projects ... and a few achievements. In this context of dynamic peri-urban areas, the proposed communication examines the role of actors (political office, AOT, operators, manufacturers, ...) and focuses on decision makers. It proposes to examine this issue based on concrete examples. First, from the characteristics and specificity of the accomplishments of tram-train technology, we examine the process of their implementation as well analysis of those remained in the draft stage. Then we focus more specifically to the many actors and their cooperation to meet the needs of coordination. Finally, meet the challenges of the territories, it is interesting to observe that they are the prospects for development.

\section{INDEX}

Mots-clés : territoire, mobilité, autorité organisatrice, interconnexion, exploitation

Keywords : territory, mobility, operating authority, interconnection, operation 
AUTEUR

JOËL FORTHOFFER

Ingénieur commercial SNCF, commission de géographie des transports.

joel.forthoffer[chez]numericable.com 